\title{
Local scour and flow characteristics around a circular cylinder undergoing vortex-induced vibration
}

\author{
S.-C. Hsieh, Y.-M. Chiew \& M.-X. Wei \\ School of Civil \& Environmental Engineering, Nanyang Technological University, Singapore \\ Y. M. Low \\ Department of Civil \& Environmental Engineering, National University of Singapore, Singapore
}

\begin{abstract}
Vortex-induced vibration (VIV) has been extensively studied and the related findings presented in published literature. However, when the cylinder is placed near an erodible sand bed, interactions between the vibrating cylinder, flow field and local scour evidently will become very complex. The aim of this study is to provide an improved understanding of the interaction between the flow field, free vibrating cylinder and scour by using flow visualization and a new PIV measurement technique. The results show that the amplitude and frequency of the vibrating cylinder are closely related to the depth of the scour hole. Based on the qualitative observation and quantitative measurement of the flow field, vibrating cylinder and scour dimensions, three distinct scour stages are identified in this study. The characteristics of the turbulence intensity, formation and transmission of the vortices in each of these three scour stages are discussed in this paper.
\end{abstract}

\section{INTRODUCTION}

Submarine pipelines laid on an erodible seabed are used extensively to transport hydrocarbons and other fluids in the offshore industry. As currents flow past the pipeline, the phenomenon, known as vortexinduced vibration (VIV), and scour around the pipeline often are observed. Interactions between the vibrating pipeline, flow field and scour bed are very complex, and can cause damage to the pipeline. Because of this, pipeline failure, which is an important topic in pipeline design, has attracted extensive research attention for many decades.

Vortex-induced vibration (VIV) near a plane boundary has been extensively studied and the related findings presented in published literature. However, most of the previous researches mainly study the response amplitude $A^{*}$ (where $A^{*}=A / D, A=$ vibration amplitude, $D=$ cylinder diameter) with different reduced velocity $V_{r}$ (where $V_{r}=u_{0} / f_{N} D, u_{0}=$ free stream velocity, $f_{N}=$ natural frequency in fluid) and the gap ratio $G / D$ (where $G=$ net gap between the surface of circular cylinder and plane wall). Fredsøe et al. (1985) considered a cylinder mounted elastically near a rigid plane wall and observed that the vibrating characteristics of the cylinder would be affected when $G / D<1.0$. Yang et al. (2009) investigated the amplitude and frequency responses of a vibrating cylinder near a rigid plane boundary with different $G / D$, and found that there is a clear difference between large $(G / D>0.66)$ and small $G / D(G / D<$ 0.3). Wang et al. (2013) used PIV (particle image ve- locimetry) to investigate the effect of a rigid planar boundary on the vortex formation, and classify the wake flow patterns into three regimes as function of $G / D$ : (1) vortex-shedding suppression region $(G / D<$ 0.3 ), where the vortex shedding occurs periodically only in the upper shear layer and forming a singlerowed vortex street; (2) intermediate region $(0.3 \leq$ $G / D<1.0$ ), where the wall effects are significant resulting in flow asymmetry about the cylinder centerline; and (3) wall-effect-free region $(G / D \geq 1.0)$, where the flow resembles that of a free-standing cylinder.

There are limited studies pertaining to the study of the interaction between vibrations of a submarine pipeline and the resulting scour on an erodible seabed. Ye (1987) investigated the scouring effect of pipe vibrations near an erodible sand bed and observed the characteristics of the pipe vibration during the sagging process of the pipeline. He observed that the initial stage of the scour beneath the pipeline was relatively slow. When $G / D=0.7$, the pipeline started its sustained vibration with appreciable amplitude, and the scour hole was eroded much quicker and deeper. Gao et al. (2006) also found that the vibrating cylinder may induce a deeper scour hole than a fixed cylinder for a given value of initial $G / D$. Recently, Li et al. (2013) conducted experiments to study the impact of a vibrating SCR on scour in a steady current. A forced vertical vibration of the riser was used in the tests. The results showed that the scour profiles for a stationary and a vibrating riser were different. The maximum scour depth increased 
significantly with both vibration amplitude and frequency, with the latter having a more profound influence.

In the limited published studies pertaining to this phenomenon, most of them had focused on the effect of scour and the response amplitude of the vibrating cylinder with different gap ratios $G / D$. There is as yet no detailed quantitative study on the flow field that is essential for a thorough understanding of the underlying physics. This is partly due to the limitations of the flow field measurement technique. The aim of this study is to provide an improved understanding of the interaction between the flow field, free vibrating cylinder and scour by using flow visualization and a new PIV measurement technique. The present work will only focus on the intermediate region $(G / D=0.45)$, which is the most interesting in the context of VIV near a boundary. To better understanding the scour process, a new high resolution PIV system was applied. This new PIV system combines the high speed camera with a continuous-wave laser to accurately measure both the mean velocity and the turbulence characteristics. In addition, the variations of vibrating amplitude and frequency, and the bed scour mechanics are also measured in the study.

\section{EXPERIMENTAL SETUP}

In order to obtain a better understanding of the process of scouring by VIV, the experiment only focuses on one significant case, namely at $G / D=0.45$. The experimental setup and PIV measured technique are described in the following section.

\subsection{Water flume, sand bed, test model and coordinate system}

The experiment was performed in a re-circulating flume that was $11.0 \mathrm{~m}$ long, $0.6 \mathrm{~m}$ wide and $0.6 \mathrm{~m}$ high; it has glass walls on both sides and the bottom to allow visual observations and PIV measurements. A honeycomb structure of length $0.2 \mathrm{~m}$ is located at the entrance of the flume to remove any large-scale irregularities in the inlet flows. One uniform sand with median grain size $d_{50}=0.449 \mathrm{~mm}$ and geometric standard deviation $\sigma_{g}=1.296$ was used. The sand bed was $8.4 \mathrm{~m}$ long with a thickness of $10 \mathrm{~cm}$. The pipeline model was placed at a distance $7.0 \mathrm{~m}$ downstream the inlet section of the sand bed.

In the test section, a constant approach flow with a velocity $u_{0}=19.6 \mathrm{~cm} / \mathrm{s}$ (outside the bottom boundary layer) was used and the water depth on the undisturbed sand bed level was maintained at $0.4 \mathrm{~m}$. The free-stream flow is uniform and the turbulence intensity is less than $1.2 \%$. The experiment was conducted under a clear-water scour condition with the critical velocity ratio $U / U_{c}=0.73$, in which $U_{c}$ was calculated by using the Shields diagram and the mean flow velocity for a rough bed.

An acrylic circular cylinder of diameter $0.035 \mathrm{~m}$ and length $0.56 \mathrm{~m}$ was used as the model in the experiments. The cylinder was mounted on an aluminum supporting frame, which is connected by two springs to a frame fixed to the flume, as illustrated in figure 1 . The supporting frame is free to vibrate only along the vertical direction due to the presence of four bearings. The total weight of the vibrating system, which included the circular cylinder and the aluminum frame was $1.445 \mathrm{~kg}$, corresponding to an equivalent mass ratio $m^{*}=2.682$. After testing free decay in still water by following the procedure in Sumer and Fredsøe (1997), several characteristic parameters were obtained: natural frequency in fluid $f_{\mathrm{N}}$ $=0.847 \mathrm{~Hz}$, damping ratio $\zeta=0.04$, and $m * \zeta=$ 0.107 .

Figure 2 shows the coordinate system used in this study. The origin is located on the undisturbed sand bed level and directly beneath the center of the cylinder. The $x$-axis is in the streamwise direction, while the $y$-axis in the vertical direction.

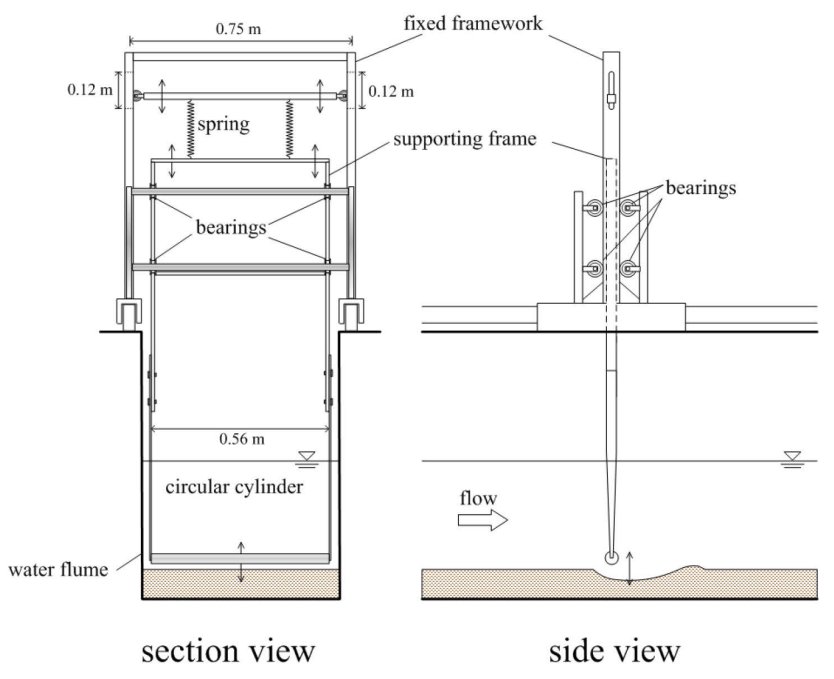

Figure 1. Schematic diagrams of experimental setup.

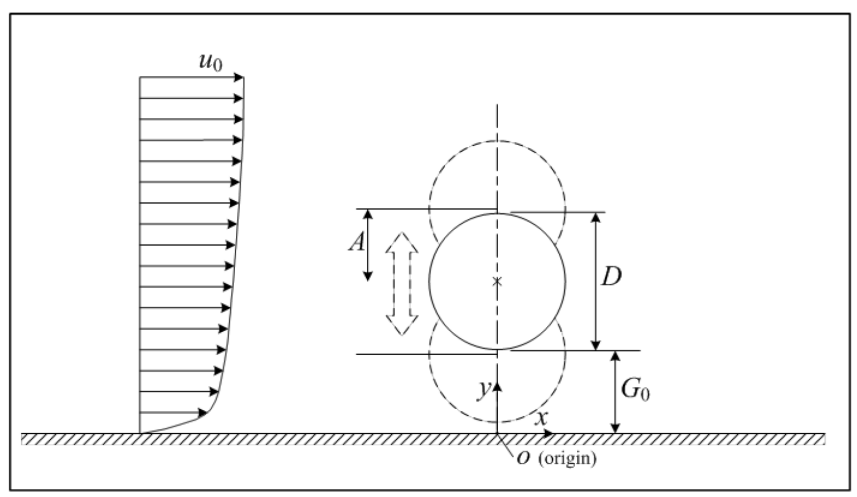

Figure 2. Coordinate system adopted in present study.

\subsection{Velocity measurements and analysis techniques}

The PIV measuring system comprised a $4 \mathrm{~W}$ aircooling laser with wavelength $=532 \mathrm{~nm}$ as the light source, and a high speed camera (Phantom Miro 
M120) to capture the images. The beam emitted from the laser source was reflected by a mirror, resulting in a laser light fan with $1.5 \mathrm{~mm}$ thickness cast downward into the water through a transparent acrylic sheet on the water surface, which reduced the reflection from water surface oscillations. Additionally, aluminum particles with diameter $=10 \mu \mathrm{m}$ and specific density $=2.7$ were used as seeding particles in the experiment. Using Stoke's law, the settling velocity of the aluminum particles was estimated to be less than $10 \mu \mathrm{m} / \mathrm{s}$, which is much smaller than the free stream velocity $u_{0}$.

The high speed camera with three-gigabyte memory storage and $1600 \times 1200$ pixel resolution was used to capture images of the particle-laden flow. A $60-\mathrm{mm}$ focal lens was mounted on the high speed camera which was operated at $200 \mathrm{fps}$ (frame per second). A multi-grid processing algorithm was adopted to enhance the accuracy of velocity calculation; the interrogation window started from $32 \times 32$ pixels and ended with $8 \times 8$ pixels.

\subsection{Image processing}

Although PIV techniques and related applications have become mature in the past decade, there are still some limitations that need to be resolved for the present study. Particularly, the accuracy of velocity measurement is insufficient in the vicinity of the cylinder or sand bed boundary due to the disturbance from the reflection and scattering of the laser light. To this end, Hsieh's (2008) proposed digital image high-pass filter technique was used to reduce reflections and scatterings of the laser light, thus enhancing the signal strength of each particle in the captured image.

In the frequency domain of a digital image, low frequency signals usually signify smoothly changing light signals from the surroundings, such as reflection or scattering from an object boundary. On the other hand, the object outline where light intensity varies sharply, such as the object boundary or light points reflected by particles, belongs to the high frequency portion. For the reason, choosing a suitable high-pass filter function can reduce or filter out low frequency signals (reflected or scattered light). The Laplacian filter function is adopted because it not only can reduce low frequency signals but also enhance high frequency signals. Eq.(1) shows the Laplacian equation of $f$,

$$
\nabla^{2} f=\frac{\partial^{2} f}{\partial x^{2}}+\frac{\partial^{2} f}{\partial y^{2}}
$$

Additionally, the image processing method for reducing body boundary effects (Cowen and Monismith 1997) was also applied.

In addition, a multi-time interval method is used to improve the defect arising from using only single time intervals to calculate particle displacements between two subsequent images. Details of this PIV technique can be found in Hsieh (2008).

\section{RESULTS}

The purpose of this study is to highlight the characteristics of the scour process and interaction between a vibrating cylinder and seabed scour. It is found that the scour process can be divided into three primary stages; the detailed results are presented below.

\subsection{Characteristic of velocity field around cylinder}

Figures 3 and 4 show the results of the visualization and PIV measurement at 120 minutes after the commencement of the test. The phase-averaged analysis was used in the velocity fields, and the dimensionless vorticity contour superimposed in figure 4. Herein, the dimensionless vorticity, which is defined as $\omega_{z} D / u_{0}$, in which $\omega_{z}$ $(=(\Delta v / \Delta x)-(\Delta u / \Delta y))$ is the spanwise vorticity of the flow field. The red and blue vorticity contours denote positive vorticity (in the counter-clockwise direction) and negative vorticity (in the clockwise direction), respectively. Moreover, it is assumed that the displacement of the cylinder, $\eta$ is sinusoidal, and can be represented by $\eta(t)=A \cos (t / T)$, where $T$ is the vibrating period of the cylinder, and the time $t$ varies from 0 to $T$.

In the figures, two scour holes and one sand dune are clearly observed. The spatial variation of the scour holes and sand dune is important for the flow pattern and scour process around the vibrating cylinder. For this reason, the geometric parameters between these scour holes and dune are schematically defined in figure 5, in which the scour hole that forms directly beneath the vibrating cylinder is called scour hole $S_{1}($ at $x / D \approx 0.0)$ and that forms farther downstream is called scour hole $S_{2}$, (at $x / D \approx$ 1.0).

Figures 3(a)-(d) show the flow pattern at $t=0$, $0.17 T, 0.33 T$ and $0.5 T$, respectively, during which the cylinder was moving toward the sand bed $(\eta / A=$ 1 to -1$)$. Throughout this period, the gap between the cylinder and sand bed decreases, and a gap flow that resembles a jet passes beneath the cylinder toward the downstream direction, as is also illustrated in figures 4(a)-(d). Meanwhile, the jet induces a counter-clockwise vortex downstream of the cylinder. When the cylinder starts to ascend, this counterclockwise vortex impinges onto the sand bed at scour hole $S_{2}$, before convecting downstream along the sand bed, as show in figures 3(e-f) and figures 4(e-f). At this juncture, some sand in scour hole S2 is suspended and transmitted downstream toward the sand dune. 
(a)

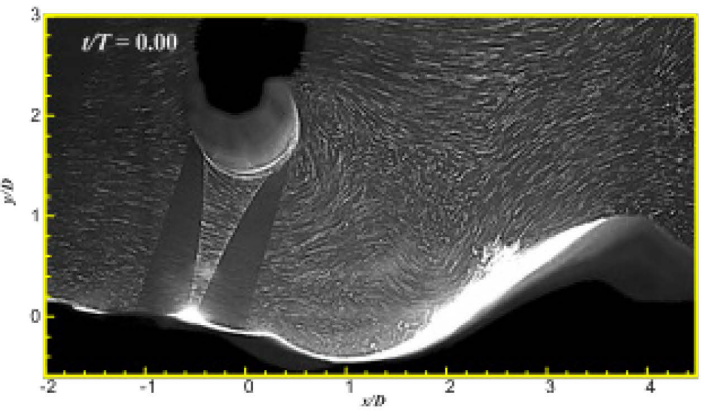

(b)

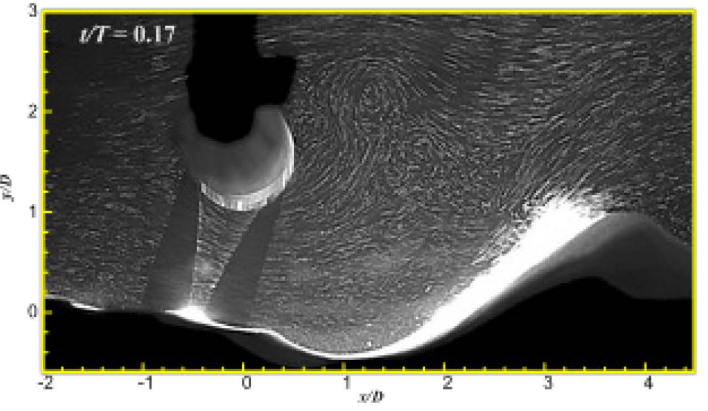

(c)

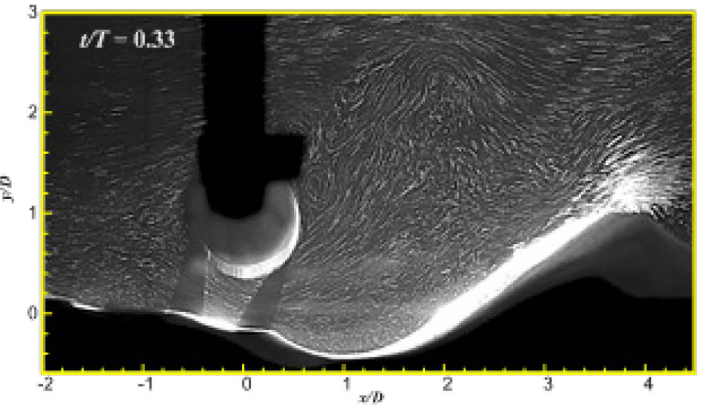

(d)

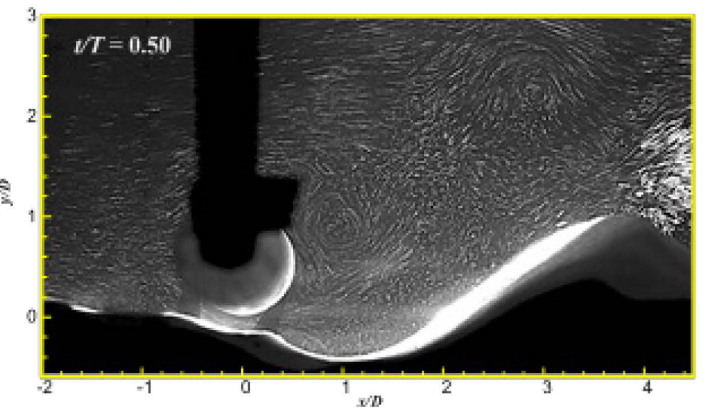

(e)

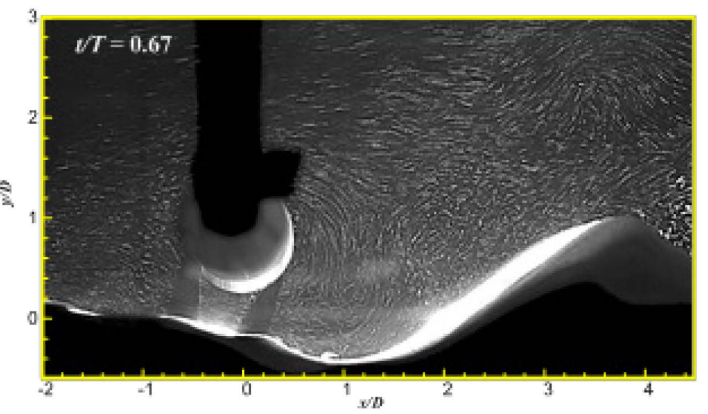

(f)

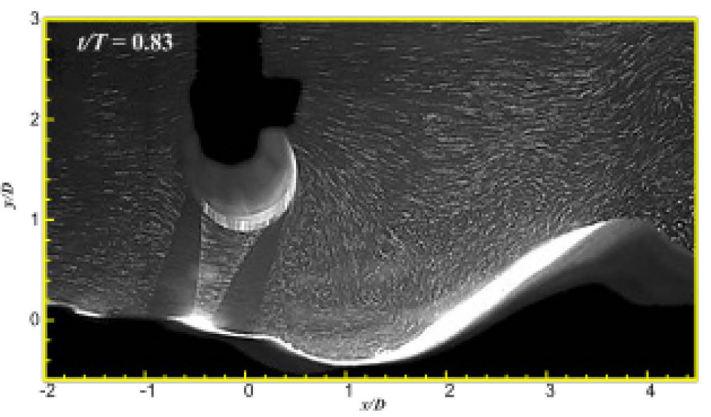

Figure 3. The result of the visualization of a free vibrating circular cylinder induced bed scour. (a)

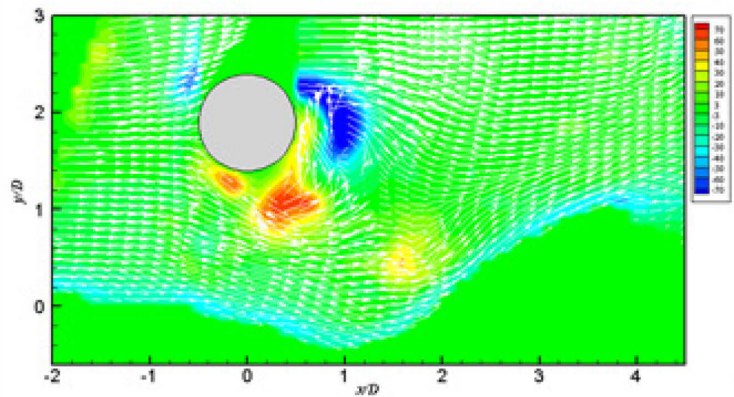

(b)

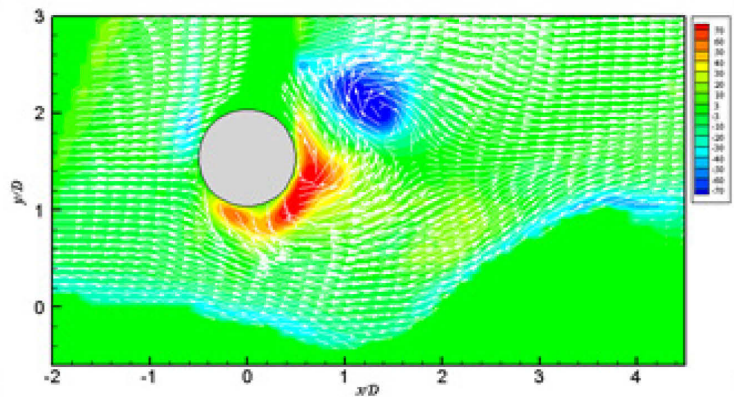

(c)

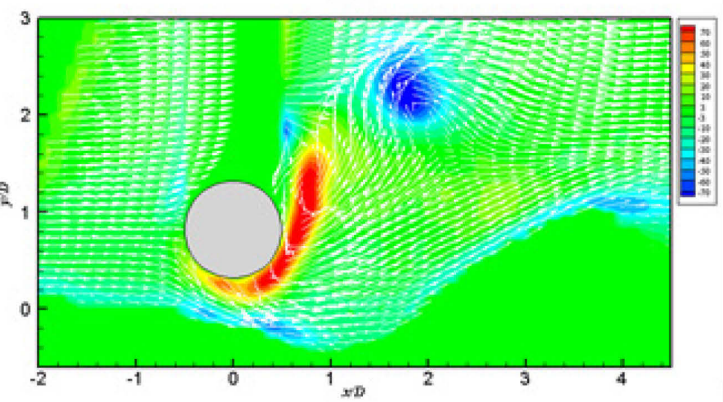

(d)

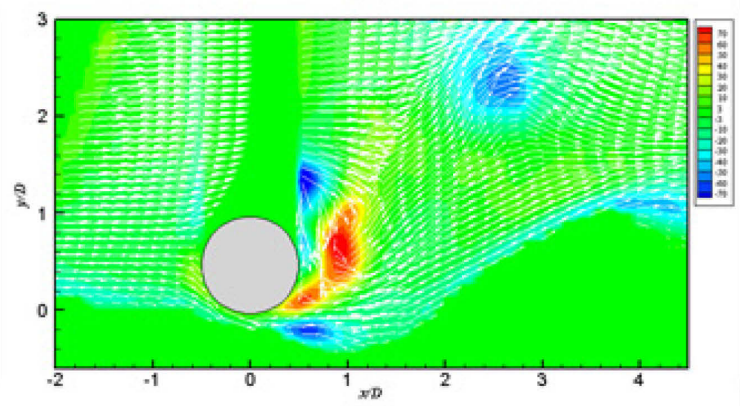

(e)

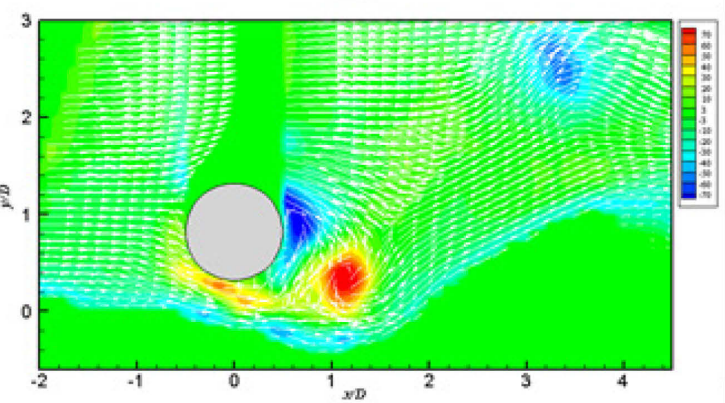

(f)

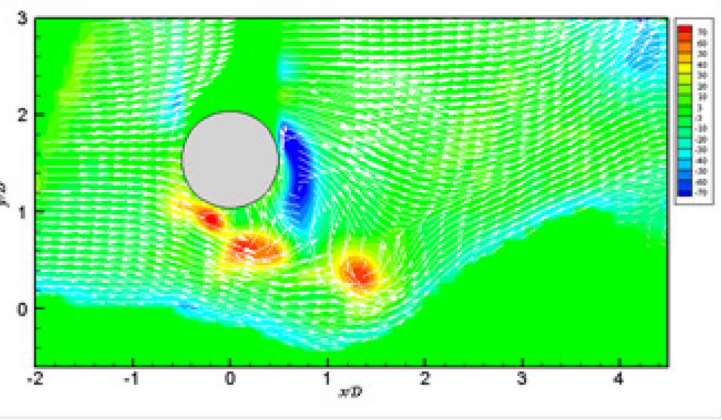

Figure 4. The result of the PIV measurement of a free vibrating circular cylinder induced bed scour. 


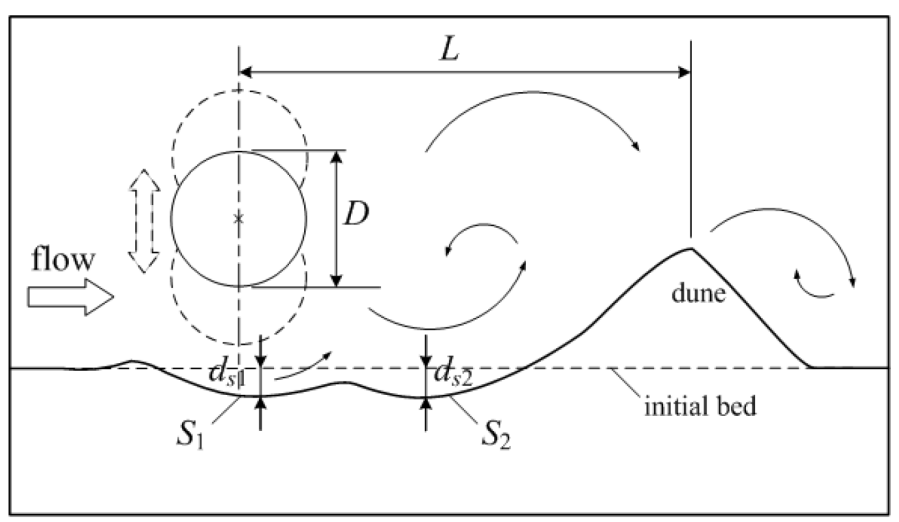

Figure 5. Schematic definition of the geometrical parameters.

Based on the visualization and PIV measurement, two primary mechanisms that accounts for scouring can be identified. First, the flow beneath the cylinder is subjected to a "compress-release" mode, somewhat resembling that of a piston. This periodic motion has a very important influence on the normal stresses, and enhances sediment transport or scour on the sand bed beneath the cylinder (scour hole $S_{1}$ ). During the ascending phase of the cylinder, the bed particles are lifted to be entrained downstream by the jet that forms when the cylinder descends. Second, as the shed vortex induced by the vibrating cylinder moves along the sand bed, it causes the formation of a second scour hole $\left(S_{2}\right)$ downstream of scour hole $S_{1}$. The moving vortex, which is present at $x / D>\approx$ 1.0 , clearly would be responsible in transporting the ejected sediment particles farther downstream, to be deposited at the sand dune.

\subsection{Mean velocity and turbulence characteristics in different scour stage}

The purpose of this section is to highlight the relationship between the mean velocity field, turbulence characteristics and sand bed scouring associated with a vibrating cylinder. The results reveal that interactions between the scour hole formation and cylinder vibrations for the entire scour process can be divided into three stages.

\subsubsection{Initial stage of the scour process}

The initial stage of the scour process refers to the period in which the depth of scour hole $S_{1}$ is larger than or equal to that of scour hole $S_{2}$, i.e., $d_{s 1} \geq d_{s 2}$, as shows in figure 6 . In this scour stage, the cylinder just starts to vibrate and the sand bed remains essentially flat at most location. The primary scour mechanism is related to the piston-like "compressrelease" mode induced primarily by the vibrating cylinder.

Figure 7 shows the measured PIV results of the mean velocity field and turbulent properties in initial stage $(t \approx 6.1 \mathrm{mins})$. The turbulence properties exemplify the velocity fluctuations in the horizontal and vertical directions, as shown in figures 7(a) and 7(b), respectively.

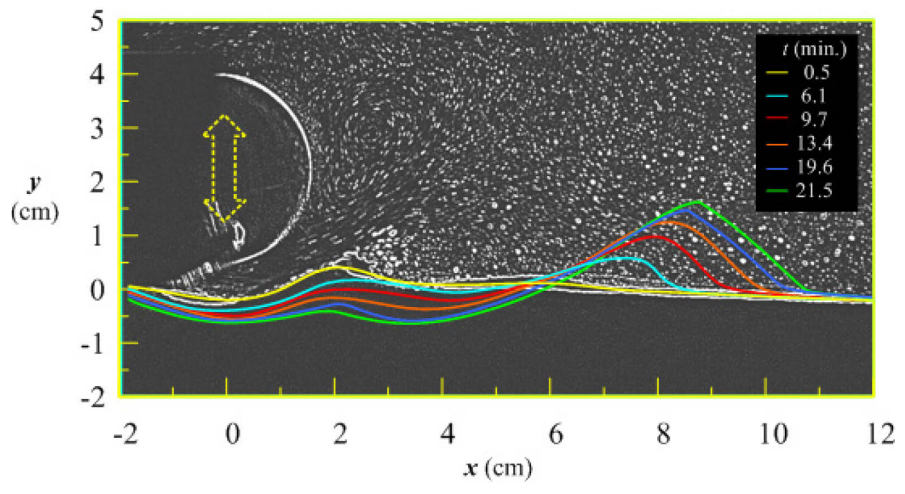

Figure 6. The variations of the sand bed in initial stage of the scour process.
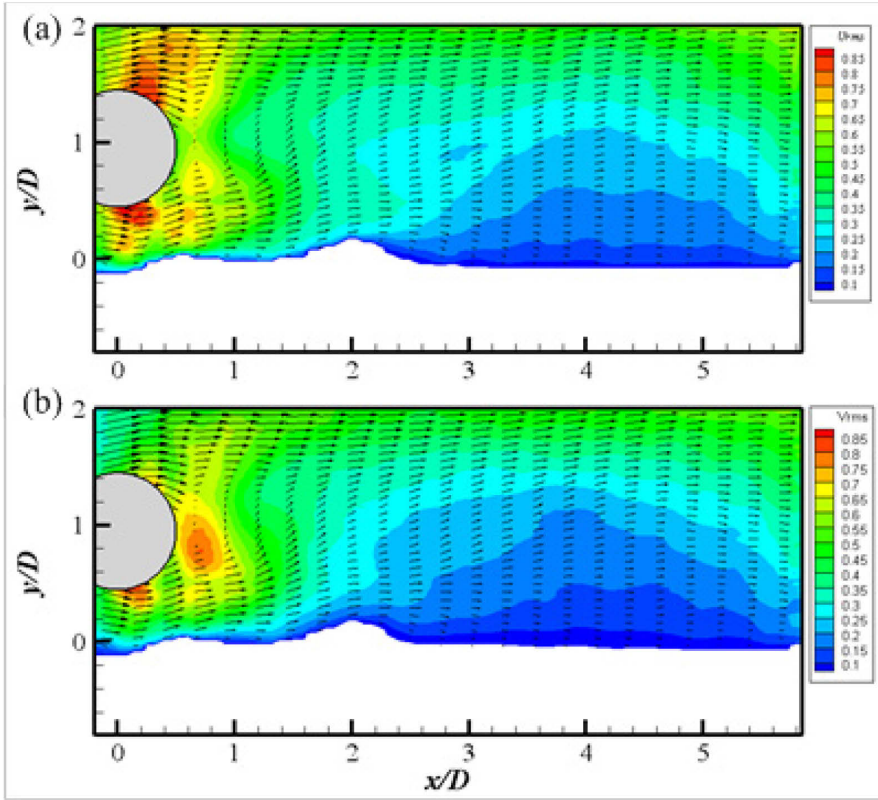

Figure 7 . Mean velocity field and turbulent properties, at $t \approx 6.1$ mins. (a) horizontal; (b) vertical.

The mean velocity field exhibits how the gap flow beneath the cylinder act somewhat like a jet flow directed upward along the scour hole $S_{1}$ boundary, before expanding and diffusing when it reaches the scour hole $S_{2}$. It may be inferred from the result that the scour mechanism related to the gap flow is stronger than the shed vortex during this stage of scour development. Moreover, the turbulence intensities are weaker around scour hole $\mathrm{S}_{2}$, especially the vertical fluctuation (see figure $7 \mathrm{~b}$ ). This phenomenon explains that only the horizontal shear stress causes scouring at scour hole $S_{2}$, In other words, the effect of the shed vortex induced by the vibrating cylinder is not significant at the initial stage.

\subsubsection{Second stage of the scour process}

When the scour hole $S_{1}$ becomes deeper, the response amplitude of the cylinder vibration correspondingly increases. In this process, the strength of the shed vortex induced by the vibrating cylinder and 
the scour rate of scour hole $S_{2}$ also increases with the vibrating amplitude. As the scour depth $d_{s 1}<d_{s 2}$, the scour process changes to the second stage.

Figure 8 shows variations of the sand bed in the second stage of the scour process, and figure 9 shows the results of the mean velocity field and turbulent properties at $t \approx 191$ mins. In this scour stage, variations of the scour depth of scour hole $S_{2}$ is distinctly bigger than $S_{1}$. Moreover, the length of $S_{2}$ also expands with the scour depth.

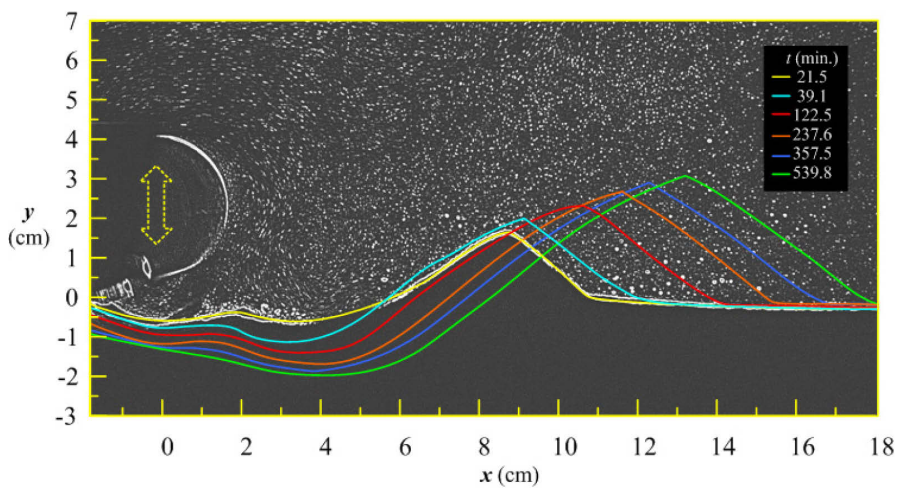

Figure 8 . The variations of the sand bed in second stage of the scour process.
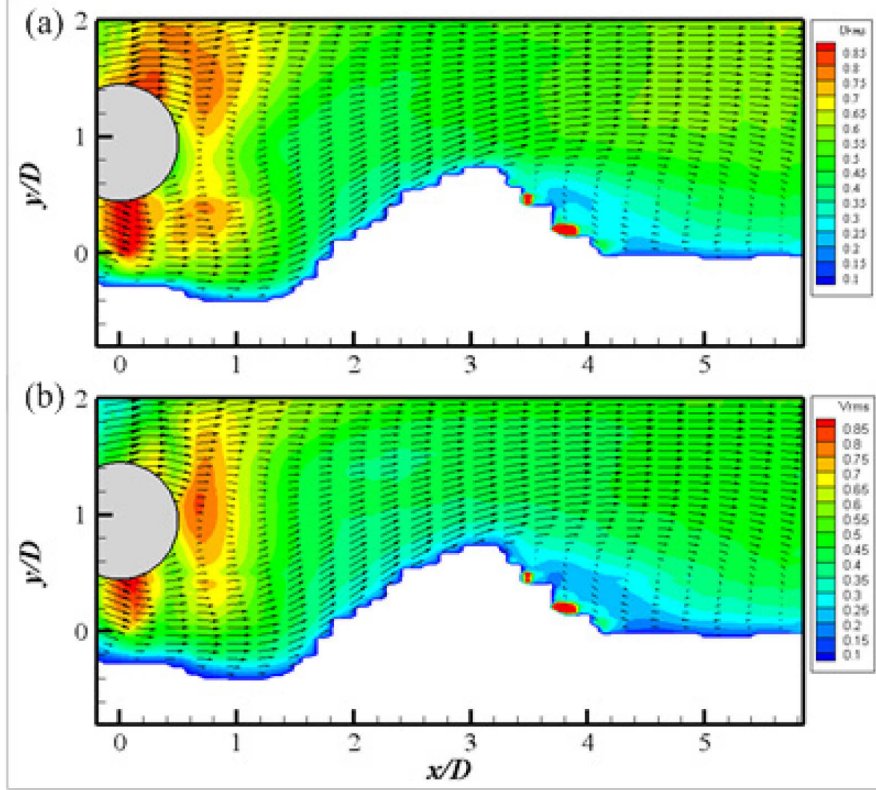

Figure 9. The results of the mean velocity field and turbulent properties, about $t=191$ mins. (a) horizontal direction; (b) vertical direction.

The result of the mean velocity field shows that the gap flow beneath the cylinder, which is now directed downward, flows along $S_{2}$. In contrast with figure 4 , the shed vortex induced by the lower shear layer impinges and convects toward scour hole $S_{2}$ in the second stage, causing the turbulence intensity to be stronger than the other stages, as shows in figure 9.

\subsubsection{Third stage of the scour process}

Figure 10 and figure 11 show variations of the sand bed and the measured results of the mean velocity and turbulent properties in the third stage, respectively. When the depths of the both $S_{1}$ and $S_{2}$ increase and approached the asymptotic stage, interactions between the vibrating cylinder and sand bed becomes insignificant and the shed vortex from the lower shear layer is no longer close to the boundary of $S_{2}$. However, the dune downstream of $S_{2}$ still impedes convection of the shed vortex. Consequently, in the third stage of the scour process, the sand on the upstream end of the dune is moved by the shed vortex, causing the dune to move downstream, as shows in figure 10.

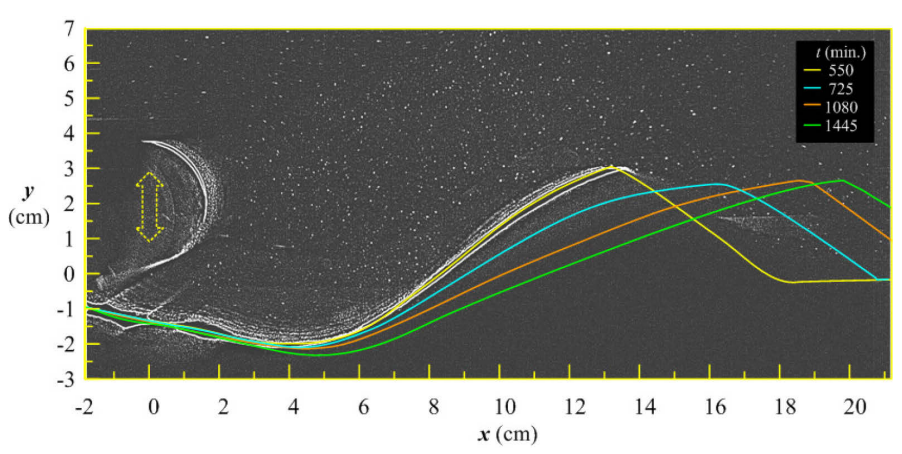

Figure 10. The variations of the sand bed in third stage of the scour process.
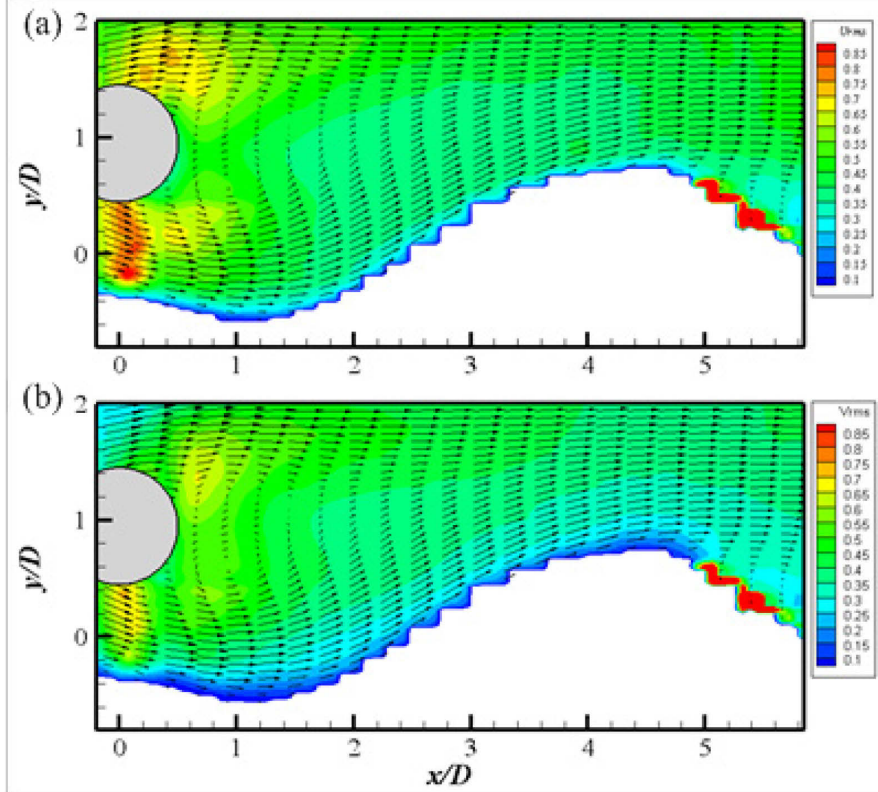

Figure 11. The results of the mean velocity field and turbulent properties, about $t=1444$ mins. (a) horizontal direction; (b) vertical direction.

\subsection{Variations of the geometric parameters of the scour hole}

Figure 12 and figure 13 show the variations of the maximum scour depth $d_{m}$ and scour length $L$ at different stages of the scour process. Figure 14 and figure 15 show the associated variations of the vibrating amplitude and frequency of the vibrating cylinder. The results reveal that that the characteristics of these parameters are closely related to the dif- 
ferent mechanism of scour. The data exhibit that dividing the scour process into three stages is necessary, because they account for the different modes of interaction between the scour, cylinder vibration and flow field.

In the initial stage, $d_{m}\left(=d_{s 1}\right)$ and $L$ increase linearly with time, while the amplitude of the vibrating cylinder likewise increases rapidly with the scour depth. The vibrating frequency, however, remains essentially unchanged. The rapid increase of the amplitude, which is closely related to the increasing scour depth at $S_{1}$, enlarges the gap between the cylinder and sand bed, allowing vortex shedding to form. As scouring the process moves into the second stage, scouring at $S_{1}$ becomes negligible and the amplitude remains essentially a constant $(A / D \approx 0.63)$. In the second and third stages, $d_{m}\left(=d_{s 2}\right)$ is the depth at $S_{2}$, and its development is related to the shed vortex. The variation of $d_{m}$ increases logarithmically, as is shown in figure 12 .

In the third stage of the scour process, the movement of the dune starts to increases significantly, causing the length of the scour hole to increase (see figure 13b). On the other hand, when the scour depth increases, the influence of the vibrating cylinder on the scoured sand bed decreases. At this juncture, the results show that the amplitude begins to decrease, and the frequency equals to a constant value $\left(f_{p}=\right.$ $0.81 \mathrm{~Hz}$ ), see figures 14 and 15 .

\section{CONCLUSIONS}

In this paper, the flow and scour characteristics of an elastically mounted cylinder undergoing VIV near a sand bed were studied experimentally using a high resolution PIV technique. For better understanding the scour process, only one case is $(G / D=0.45)$ tested because it is the most interesting in the context of VIV near a boundary. A new high resolution PIV system was applied. The main findings are summarized as follows:

1. Based on experimental observations and PIV measurements, two scour mechanisms, namely piston effect and shed vortex that cause sand bed scour are identified.

2. Based on the measured mean velocity field, turbulence characteristics, vortex behavior, and scouring process, three stages are identified in the scour process. Interactions between scouring, cylinder vibration and flow field in each of these stages are distinct.

3. During the development of the scour hole, the amplitude of the vibrating cylinder exhibits an increasing-constant-decreasing behavior in these three stages.

\section{ACKNOWLEDGMENTS}

The authors gratefully acknowledge the financial support provided by Singapore Maritime Institute (SMI) and EMAS AMC, under the SMI Deepwater Technology R\&D Programme.
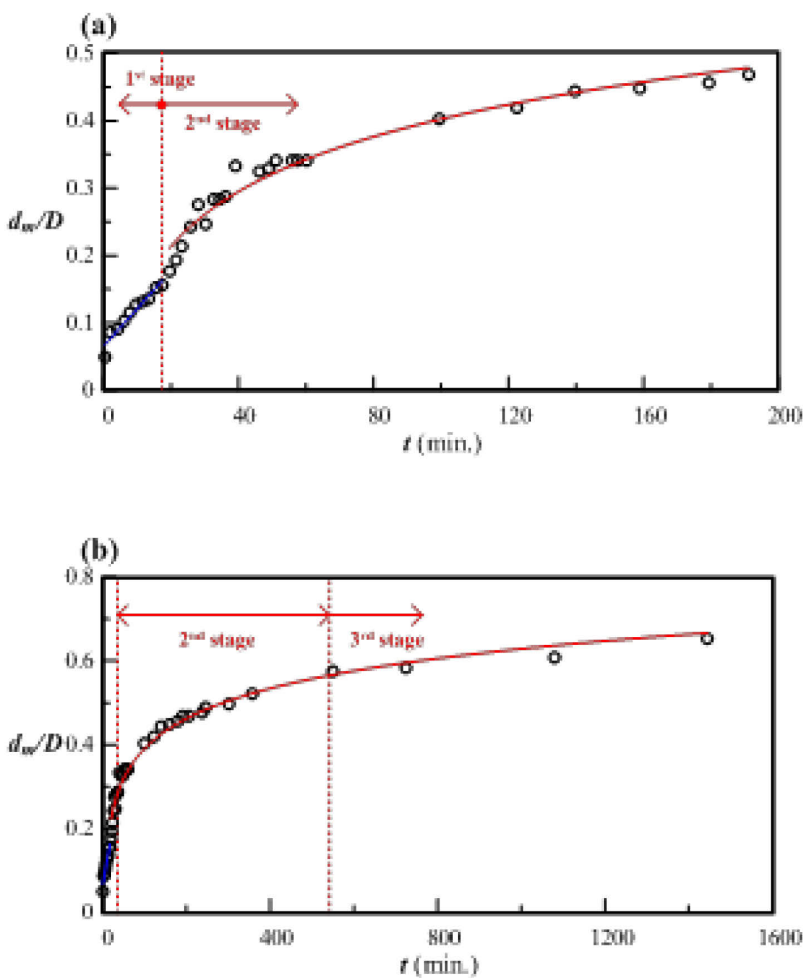

Figure 12. Variations of the maximun scour hole depth with different scour stage.

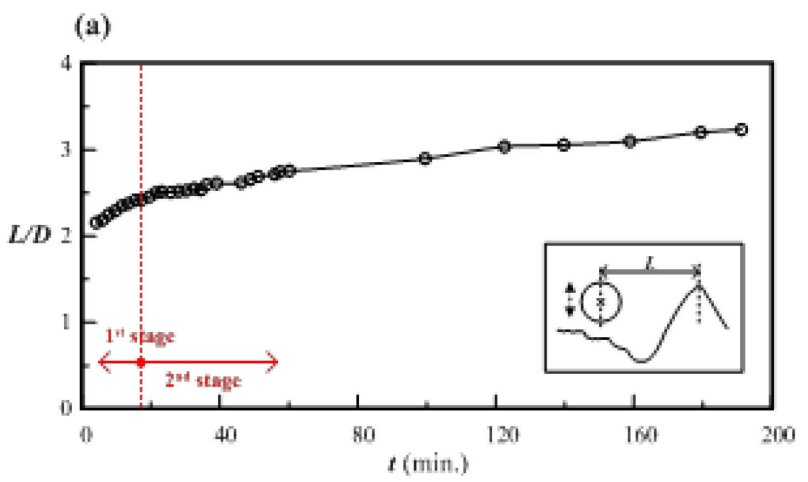

(b)

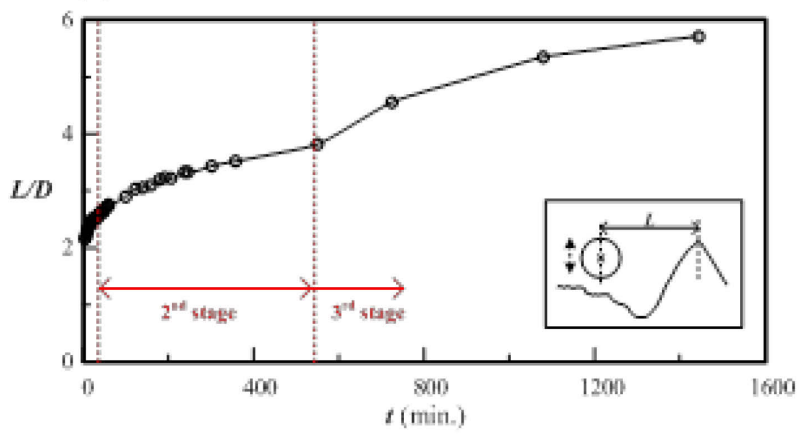

Figure 13. Variations of location of the dune with different scour stage. 

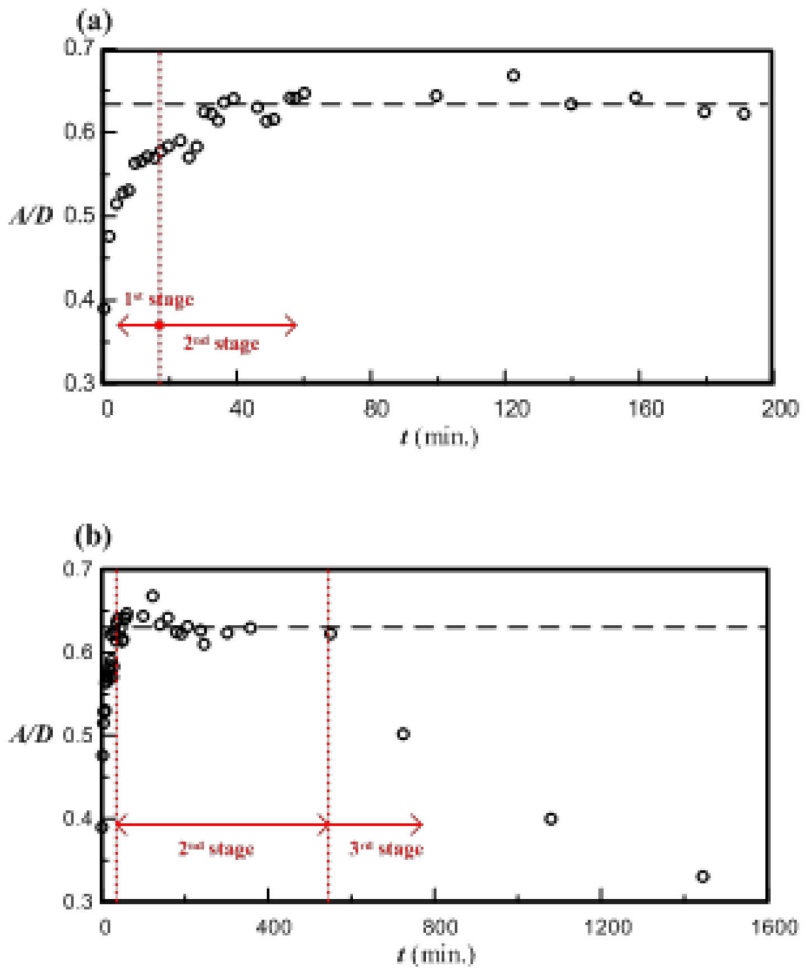

Figure 14. Variations of the dimensionless response amplitude with different scour stage.

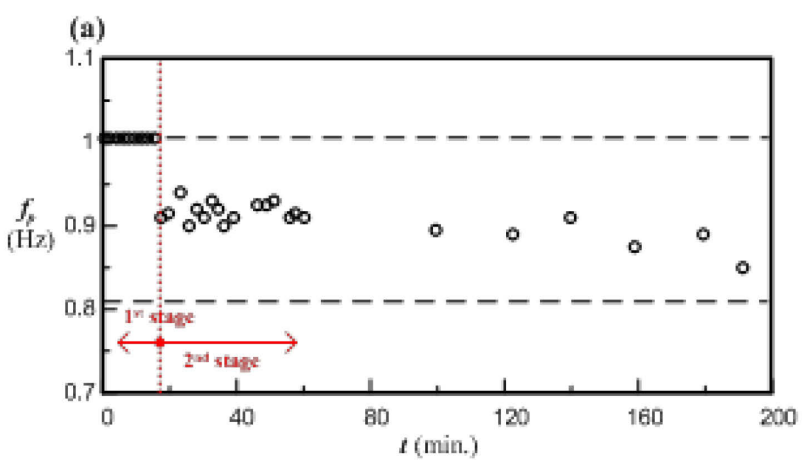

(b)

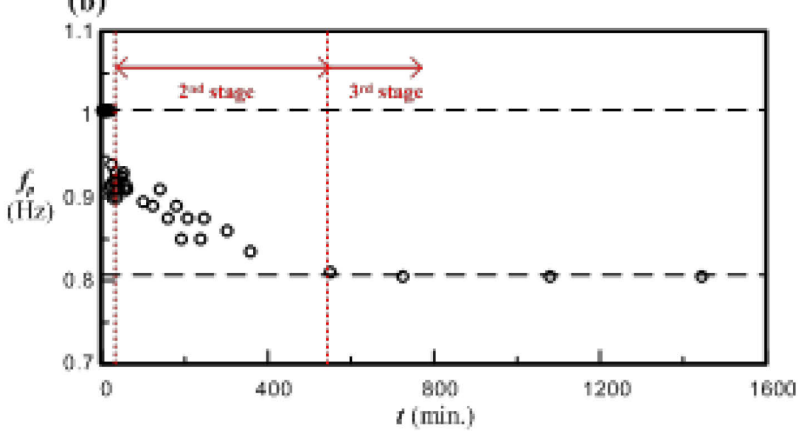

Figure 15. Variations of the vibrating frequency with different scour stage.

\section{REFERENCES}

Cowen, E.A. \& Monismith, S.G. 1997. A hybrid digital particle tracking velocimetry technique. Experiments in Fluids 22: 199-211.

Fredsøe, J., Sumer, B.M., Andersen, J. \& Hansen, E.A. 1985. Transverse vibration of a cylinder very close to a plane wall. Proceedings of the International Offshore Mechanics and Arctic Engineering Symposium 1: 601-609.

Gao, F.P., Yang, B., Wu, Y.X. \& Yan, S.M. 2006. Steady current induced seabed scour around a vibrating pipeline. $A p$ plied Ocean Research 28: 291-298.

Hsieh, S.C. 2008. Establishment of high time-resolved PIV system with application to the characteristics of a near wake flow behind a circular cylinder. Ph.D. thesis, National Chung Hsing Univ., Taiwan.

Li, F.Z., Dwivedi, A., Low, Y.M., Hong, J.H. \& Chiew, Y.M., 2013. Experimental investigation on scour under a vibrating catenary pipe. Journal of Engineering Mechanics, ASCE 139(7): 868-878.

Sumer, B.M. \& Fredsøe, J. 1997. Hydrodynamics around Cylindrical Structures. World Scientific.

Wang, X.K., Hao, Z. \& Tan, S.K. 2013. Vortex-induced vibrations of a neutrally buoyant circular cylinder near a plane wall. Journal of Fluids and Structures 39: 188-204.

Yang, B., Gao, F.P., Jeng, D.S. \& Wu, Y.X. 2009. Experimental study of vortex-induced vibrations of a cylinder near a rigid plane boundary in steady flow. Acta Mechanica Sinica 25: 51-63.

Ye, M. 1987. The flow induced pipe vibration during its sagging process. Journal of Hydraulic Research 25(5): 565582. 